\title{
Estudo Ab-initio da supercondutividade do YH3
}

\author{
Joseane S. Almeida ${ }^{\mathbf{1}}$; Álvaro S. Alves ${ }^{2}$
}

1. Bolsista PROBIC/UEFS, Graduanda em Física, Universidade Estadual de Feira de Santana, e-mail: joseanealmeida107@gmail.com

2. Orientador, Departamento de Física, Universidade Estadual de Feira de Santana, email:

asa@uefs.br

PALAVRAS-CHAVE: Supercondutividade; Método Ab-initio; YH3.

\section{INTRODUÇÃO}

Desde que a supercondutividade foi descoberta por Kamerling Onnes ao diminuir bruscamente a temperatura do mércurio, compostos tem sido investigados na expectativa de serem supercondutores de alta temperatura crítica(COSTA,2012). Atualmente existem diversos elementos químicos que alcançam esta fase ao submetêlos à pressão(DEBESSAI,2008).

Os resultados teóricos de N.W. Ashcroft(2004) sugeriram ainda que hidretos submetidos a consideráveis pressões tem a possibilidade de tornarem-se supercondutores de alta temperatura crítica. Sendo assim,numerosas investigações foram feitas, dentre elas, para hidretos de tunsgêncio, niobium e ítrio(PATRYK,2012) (GAO,2013)(LI, 2015).

O trihidreto de ítrio(YH3) possui uma gama de resultados experimentais e poucos trabalhos téoricos que consentem na transição hexagonal isolante para cúbica metálica. (ALMEIDA,2009). R. Ahuja et al., performando o cálculo DFT encontraram a fase supercondutora do YH3 na sua estrutura cúbica em 17.7 GPa a 40K(KIM,2009). Ainda existem debates sobre a estrutura desse cristal a temperatura e pressão ambiente. Os cálculos $a b$ initio de Wolf e Herzig mostram diferenças de energia muito pequenas entre as estruturas de grupos espaciais P3c1, P63cm e P63 e a análise de dados experimentais com técnicas distintas divergem entre tais estruturas. (WOLF, 2002) (FEDOTOV,2006) (KIEREY,2001). Outrora, os experimentos que investigaram o YH3 sob pressão divergiram sobre a pressão crítica entre a estrutura cristalina hexagonal e cúbica de face centrada.(OHMURA,2006)(KUME,2007).

O objetivo deste trabalho é contribuir com mais um resultado téorico, apresentando-se de acordo com as análises anteriores e indicando qual a pressão de transição para a fase cúbica supercondutora.

\section{METODOLOGIA}

Para investigar as propriedades desse sistema de muitos corpos, performamos a Teoria Funcional da Densidade(DFT). Fundamentada nos teoremas de Hohenberg e Kohn, é necessário realizar um cálculo auto-consistente, em que a precisão para a energia total foi de $10^{\wedge}-5$ Ry. Para o potencial de troca e correlação foi utilizado a aproximação da densidade local (Local Density Approximation-LDA), utilizando o método Projector augmented wave method (PAW), implementado pelo programa QUANTUM 
ESPRESSO(GIANNOZZI,2009). Com ele, os elétrons mais ligados ao núcleo são tratados por funções de onda fictícias, fazendo parte do potencial externo junto ao núcleo, enquanto os orbitais dos elétrons mais externos são expandidos em ondas planas. Na prática não lidamos com bases infinitas, desse modo escolhemos o máximo vetor de onda $(\mathrm{Km})$ associado ao cristal no espaço recíproco, tal que todos os vetores menores que ele serão empregados. O mesmo é feito para o número de pontos k usados em integrações na zona de Brillouin, em que a malha é gerada pelo método MonkhorstPack.

O segundo passo foi a otimização da estrutura cristalina. As energias totais e volumes foram fitados pela equação de estado Birch Murnaghan, onde o volume de equilíbro Vo, o bulk modulus Bo e sua derivada Bo' foram determinados para a estrutura fcc. Como o Hamiltoniano não envolve pressão, o volume de equilíbrio é tal que a pressão seja zero. Os parâmetros foram identificados através do programa SciDAVis(STUART, 2009) e com eles detemos da equação de estado do sólido.

A estrutura de bandas e a densidade de estados(DOS) permitem analisar as propriedades elétricas do YH3 e seus gráficos foram feitos no programa xmgrace.

\section{RESULTADOS E/OU DISCUSSÃO}

Os dados de convergência da energia total em função do número de pontos k e energia de corte, tratados no SciDavis, forneceram 8 e 60 Ry, respectivamente. Posteriormente, utilizando este malha de pontos 8x8x8 e energia de corte $60 \mathrm{Ry}$, otimizamos a estrutura. Os valores do parâmetro de rede, $V o, B o, B o$ ' e o gráfico com ajuste via Equação de Birch-Murnaghan são ilustrados abaixo:

Tabela 1: Otimização estrutural fcc-YH3

\begin{tabular}{|l|l|}
\hline & \\
\hline a (Bohr) & 9,12791 \\
\hline Vo (Borh) $^{3}$ & 190,13 \\
\hline Bo $(\mathrm{GPa})$ & 75,1 \\
\hline Bo & 3,55 \\
\hline
\end{tabular}

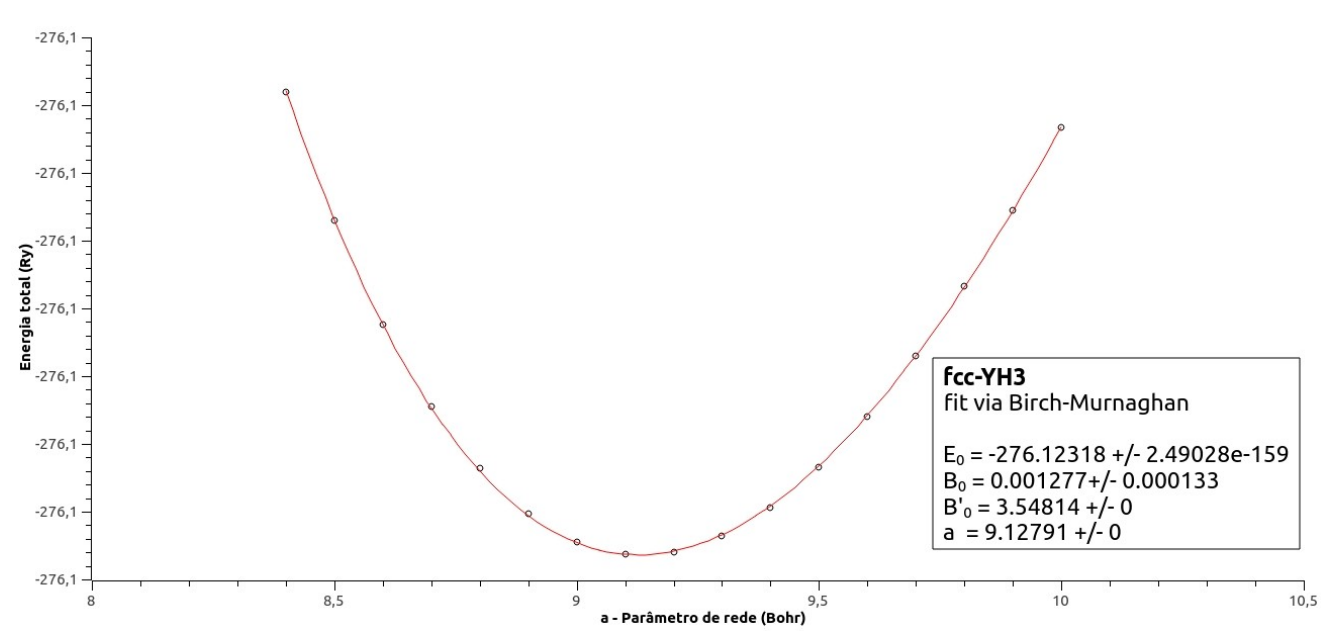

Figura 1. Otimização estrutural via equação de Birch Murnaghan 
A energia de Fermi assume o valor 12,1124 eV ao calcular a estrutura de bandas do YH3. A energia de gap é $0.0 \mathrm{eV}$, indicando caráter metálico. A densidade de estados e as bandas são representados nas figuras 2 e 3 .

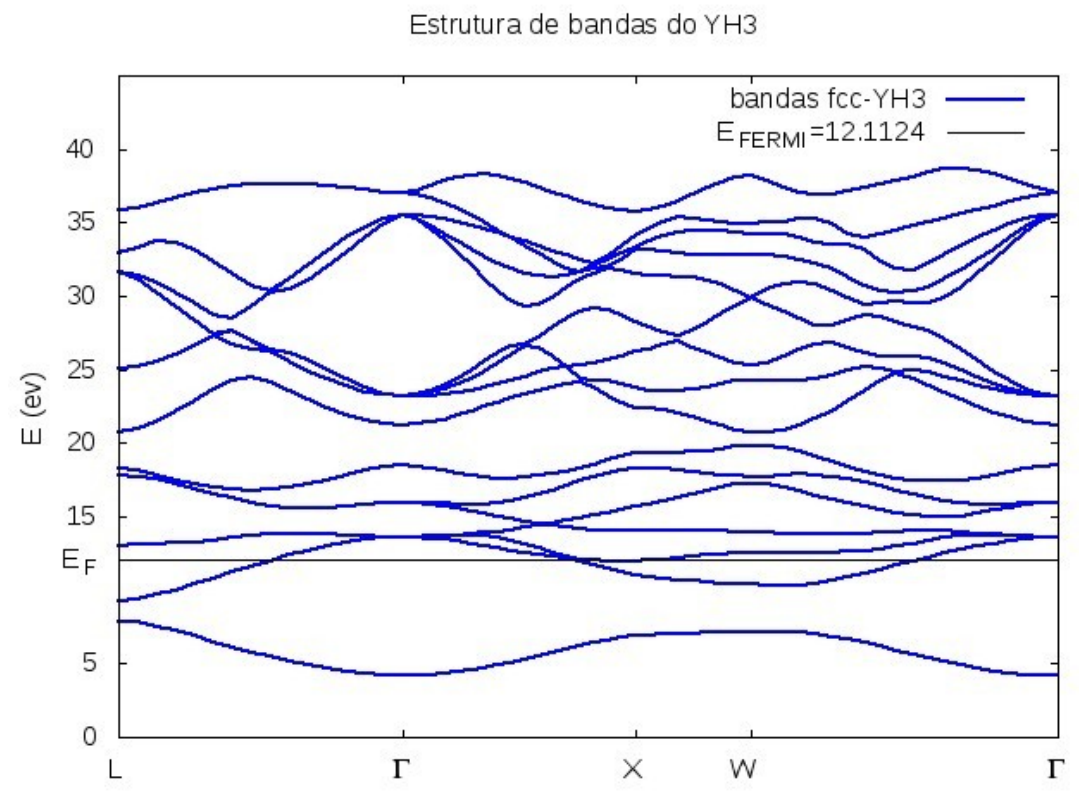

Figura 2. Estrutura de bandas do YH3

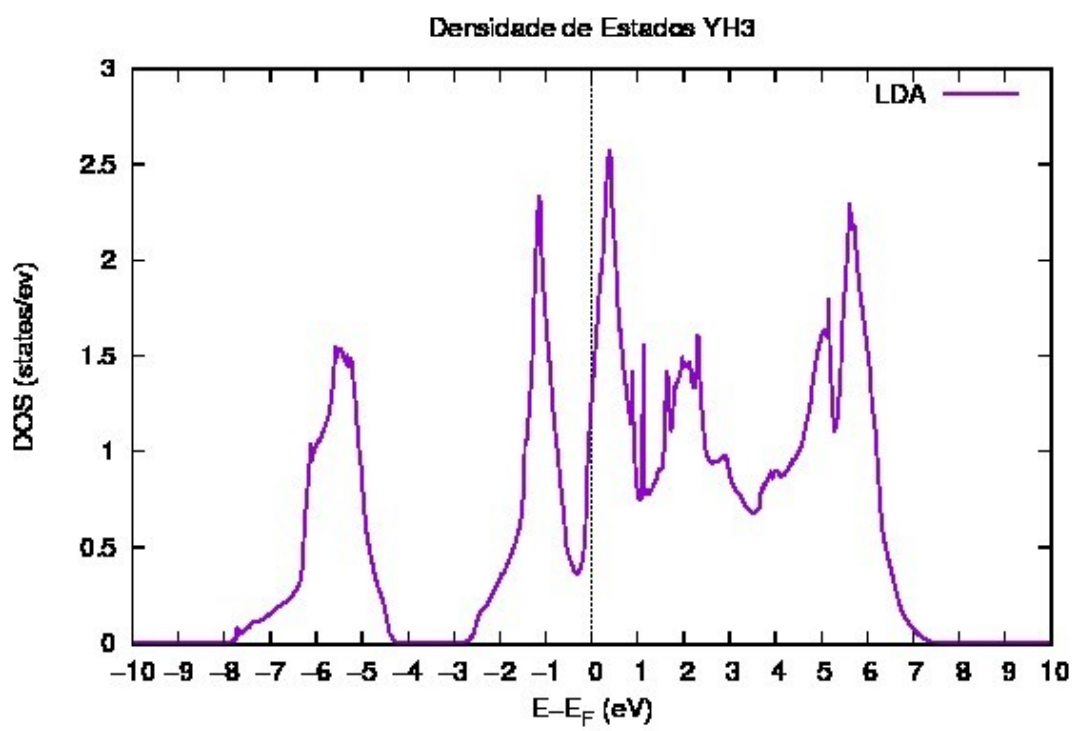

Figura 3. Densidade de estados do YH3

O estudo das propriedades estruturais e eletrônicas do YH3 na fase cúbica estão de acordo com os resultados téoricos, que afirmam o caráter métálico do composto.

Dada que nesta fase é onde a supercondutividade ocorre, os resultados obtidos serão utéis para a continuidade do trabalho. Embora houvesse a dificuldade de um bom raio muffin-tin na metodologia FP-LAPW, isto trouxe a oportunidade de testar novos códigos e novo método. 


\section{REFERÊNCIAS}

User's Guide for QUANTUM EXPRESSO, versão 3.2.

HUIBERTS, J.N; GRIESSEN R; RECTOR J.H; WIJNGAARDEN R.J; DEKKER J.P; DE GROOT D.G; KOEMAN N.J. 1996. Yttrium and lanthanum hydride films with switchable optical properties. Nature. London, 380 (231).

KIEREY, H;RODE M.; JACOB, A;BORGSCHULTE,A; SCHOENES. 2001. Raman effect and structure of $\mathrm{YH}_{3}$ and $\mathrm{YD}_{3}$ thin epitaxial films. Phys. Rev. B. 63, 134109.

WOLF, W; HERZIG. P. 2002. First-principles investigations for YH3 (YD Y $_{3}$ : Energetics, eletric-field gradients, and optical properties. Phys. Rev. B. 66, 224112.

STUART. J. 2009. LabPlot and SciDAVis Collaborate on the Future of Free Scientific Plotting.

DEBESSAI, M. et al. 2008. Comparison of the pressure dependences of TC in the trivalent d-electron superconductors Sc, Y, La, and Lu up to megabar pressures. Phys. Rev. B. 78, 064519.

ASHCROFT, N.W.2004. Hydrogen Dominant Metallic Alloys: High Temperature Superconductors? Phys. Rev. Lett. v. 92, n.18.

FEDOTOV, V.K; ANTONOV, V.E ; BASHKIN, I.O; HANSEN. T; NATKANIEC. I. J. 2006.Displacive ordering in the hydrogen sublattice of yttrium trihydride. Phys.Condens. Matter. 18, 1593-1599.

OHMURA, A; MACHIDA, A., WATANUKI, T ; AOKI, K; NAKANO, S; TAKEMURA, K. 2006. Infrared spectroscopic study of the band-gap closure in YH3 at high pressure.Phys. Rev. B. 73, 104105.

KUME, T;OHURA, H; SASAKI, S; SHIMIZU, H; OHMURA, A; MACHIDA, A; WATANUKI, T; AOKI, K; TAKEMURA, K. 2007. High-pressure study of YH3 by Raman and visible absorption spectroscopy. Phys. Rev. B. 76, 024107.

ALMEIDA, J.S; KIM, D.Y; ORTIZ, C; M.KLINTENBERG. M; AHUJA, R. 2009. On the dynamical stability and metallic behavior of YH3 under pressure. Appl. Phys. Lett. 94, 251913.

KIM, D.Y; SCHEICHER, R.H; AHUJA, R. 2009. Predicted High-Temperature Superconducting State in the Hydrogen-Dense Transition-Metal Hydride YH3 at $40 \mathrm{~K}$ and 17.7 GPa. Appl. Phys. Lett. 103, 077002.

GIANNOZZI, P et al. 2009. QUANTUM ESPRESSO: a modular and open-source software project for quantum simulations of materials. J. Phys.: Condens. Matter. 21, 395502

COSTA, M.B.S; PAVÃO, A.C. 2012.Supercondutividade: um século de desafios e super ação.Revista Brasileira de Ensino de Física, v. 34, n. 2, 2602.

PATRYK, Z.-E.; VANESSA, L.; TIMOTHY , A.S.; HOFFMANN, R.; ASHCROFT, N. W. 2012. $\mathrm{WH}_{\mathrm{n}}$ under Pressure. J. Phys.: Condens. Matter.24, 155701.

GAO, G.; HOFFMANN, R.; ASHCROFT, N. W.; LIU, H.; BERGARA, A.; Ma, Y. 2013. Theoretical Study of the Ground-State Structures and Properties of Niobium Hydrides under Pressure. Phys. Rev. B. 88, 184104.

LI, Y.; HAO, J.; LIU, H.; TSE, J.S. ; WANG, Y;MA Y. 2015. Pressure-Stabilized Superconductive Yttrium Hydrides. Sci. Rep. 5, 9948. 